\title{
Three Dimensional Image Analysis on Movement Speed Biomechanics Based on MyoResearch XP
}

\author{
Zhongmin Li \\ Department of Physical Education, Henan College of Traditional Chinese Medicine, Zhengzhou, \\ 450046, China \\ zhongmin_li@yeah.net
}

Keywords: 3D image, Relationship matrix, Logical path, Sensor, EMG, MyoResearch XP.

\begin{abstract}
In order to improve the analysis precision of muscle reaction speed in the free fight training process, this paper puts forward a new 3D image new analytic method. This method uses the relation matrix to establish analytical mathematical model of two continuous actions and obtains the logic relation path, which greatly improves the efficiency and quality of 3D image analysis. In order to verify the effectiveness and the reliability of the algorithm, this paper uses MyoResearch XP software, which has the most complete EMG and biomechanical transducer currently, to test the free fight body movement speed and the biomechanical. By testing the optimization parameters of free fight body movement speed and the biomechanical are obtained, which provides a new computer method for free fight body movement image analysis.
\end{abstract}

\section{Introduction}

In the fields of sports, free fight has characteristics of high resistance and openness. In free fight movement process, limb movement reaction speed is an important index to measure a player. Limb movement reaction speed refers to the speed of from accept stimulus to making reaction. In free fight competition process, the key of outcome is the player reaction speed; therefore the body movement reaction speed becomes the most concerned problem of athletes, coaches and scientific research workers [1-3]. This study uses the 3D image analysis technology to analyze the free fight training body movements. The basic process is as shown in Figure 1.

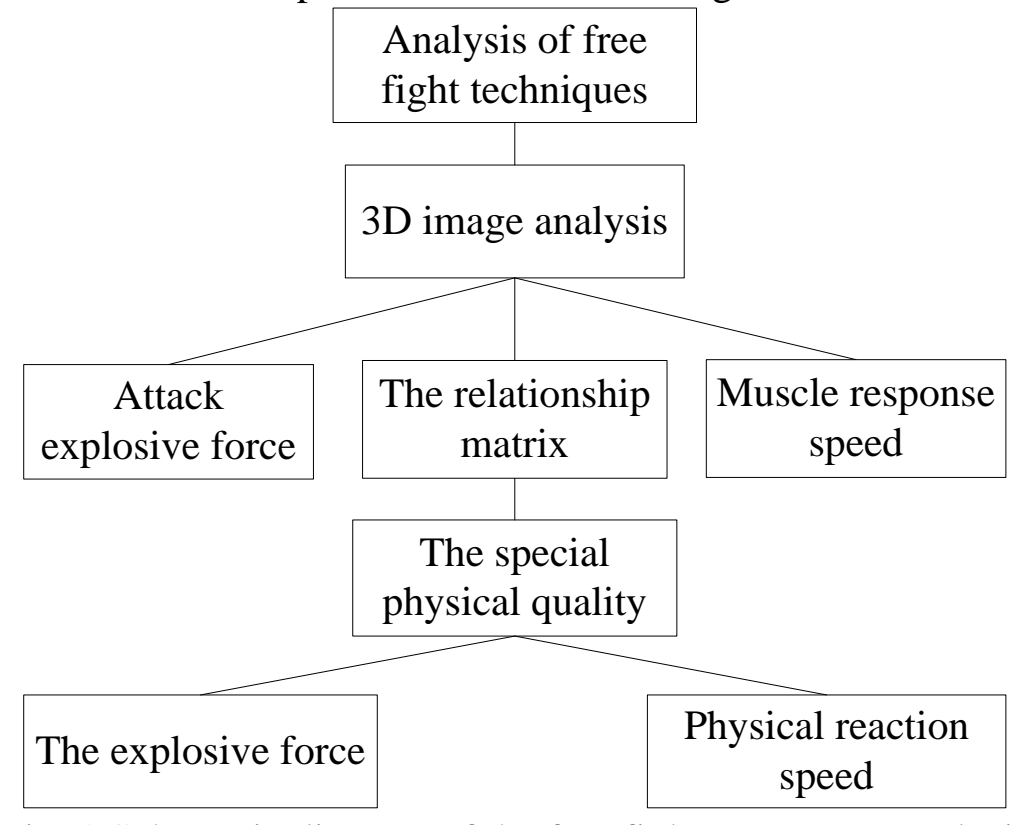

Fig. 1 Schematic diagram of the free fight movement analysis

Figure 1 shows the schematic diagram of the free fight movement analysis. From the chart, it can be seen, based on the image analysis, the free fight movement training process can obtain the muscle reaction speed and the biology explosive force [4-6]. Through the training, two parameters are optimized, finally the new reaction speed and biological explosive force are obtained. 


\section{Mathematics Model and Algorithm Design of Body Movement Image 3D Analysis}

In order to study the continuity motion speed and biomechanics of free fight 3D image, it needs to establish a 3D analytical mathematical model [7-9]. Firstly assuming continuous variable of two free fight movements $S=\left\{\mathrm{x}_{1}, \mathrm{x}_{2}, \cdots, \mathrm{x}_{\mathrm{m}}\right\}$ and $\mathrm{G}=\left\{\mathrm{y}_{1}, \mathrm{y}_{2}, \cdots, \mathrm{y}_{\mathrm{n}}\right\} . R$ is the relationship from $S$ to $G$. Firstly it needs to introduce the relation matrix.

$$
\begin{gathered}
M_{K}=\left[k_{i j}\right]_{m \times n} . \\
k_{i j}=\left\{\begin{array}{ll}
1 & \text { if }<x_{i}, y_{j}>\in R \\
0 & \text { if }<x_{i}, y_{j}>\notin R
\end{array} .\right.
\end{gathered}
$$

If there is:

$$
\begin{aligned}
& A=\{1,2,3,4\} \\
& K=\{<1,1>,<1,2>,<2,3>,<2,4>,<4,2>\} .
\end{aligned}
$$

The continuity image relation of $K$ is as shown in Figure 2.

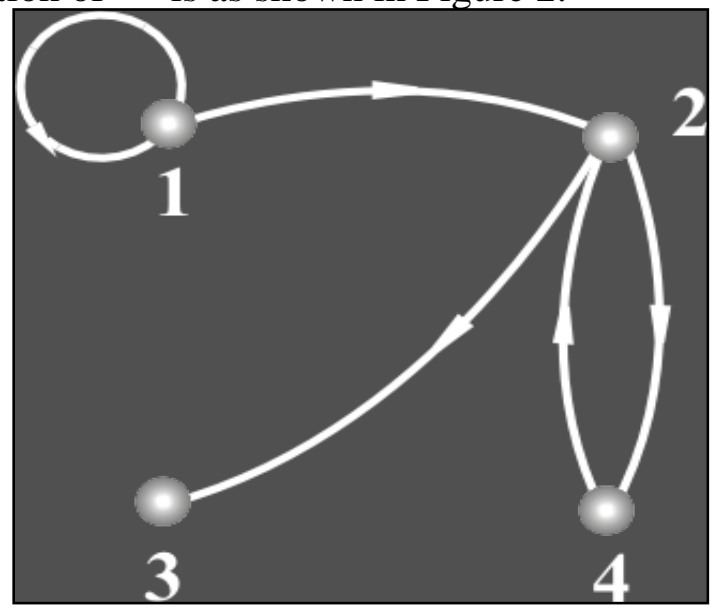

Fig. 2 Motion continuity analysis diagram

As shown in Figure 2, it is the MK logic relation path. According to the map it can read the instructions of the route, and the matrix and formula of digital signal can be got, as shown in the formula (4).

$$
M_{k}=\left[\begin{array}{llll}
1 & 1 & 0 & 0 \\
0 & 0 & 1 & 1 \\
0 & 0 & 0 & 0 \\
0 & 1 & 0 & 0
\end{array}\right] .
$$

Through the analysis of continuous actions image signal, it can judge on the semi group $\mathrm{M} 1=<\mathrm{M} 1, M 2<=<M 2,{ }^{*}>$, and $P: M 1 \rightarrow M 2$. For $x, y \in M_{1}$, there is:

$$
P(x 0 y)=P(x)^{*} P(y) \text {. }
$$

So, $\left(G_{i j}\right) n \times n$ can be called executable matrix of $D$.

According to the mathematical model of three dimensional image analysis algorithms, we can use the $\mathrm{VC}++$ program to carry on the algorithm programming, in which the main algorithms are as follows:

$\mathrm{f}=$ edge(handles.img,'canny');

imshow(f);

handles.img=f;

guidata(hObject,handles);

$\mathrm{g}=\mathrm{fftshift}(\mathrm{g})$;

$[\mathrm{M}, \mathrm{N}]=\operatorname{size}(\mathrm{g})$;

$\mathrm{nn}=2$;

$\mathrm{d} 0=80$; 


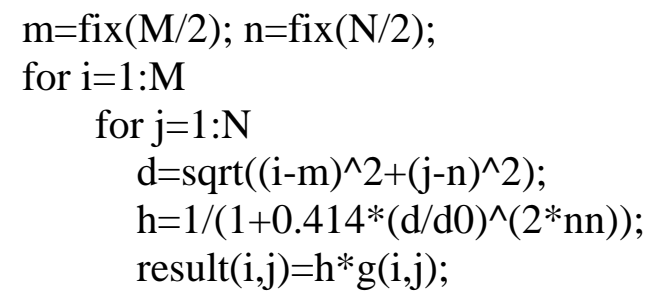

\section{The Free Fight Biomechanics Analysis based on the 3D Image}

In order to verify the effectiveness and reliability of 3D continuous motion analysis mathematical model, this study uses EMG analysis software to analyze 3D image on the free fight movement, in which the main interface of the software is shown in Figure 3.

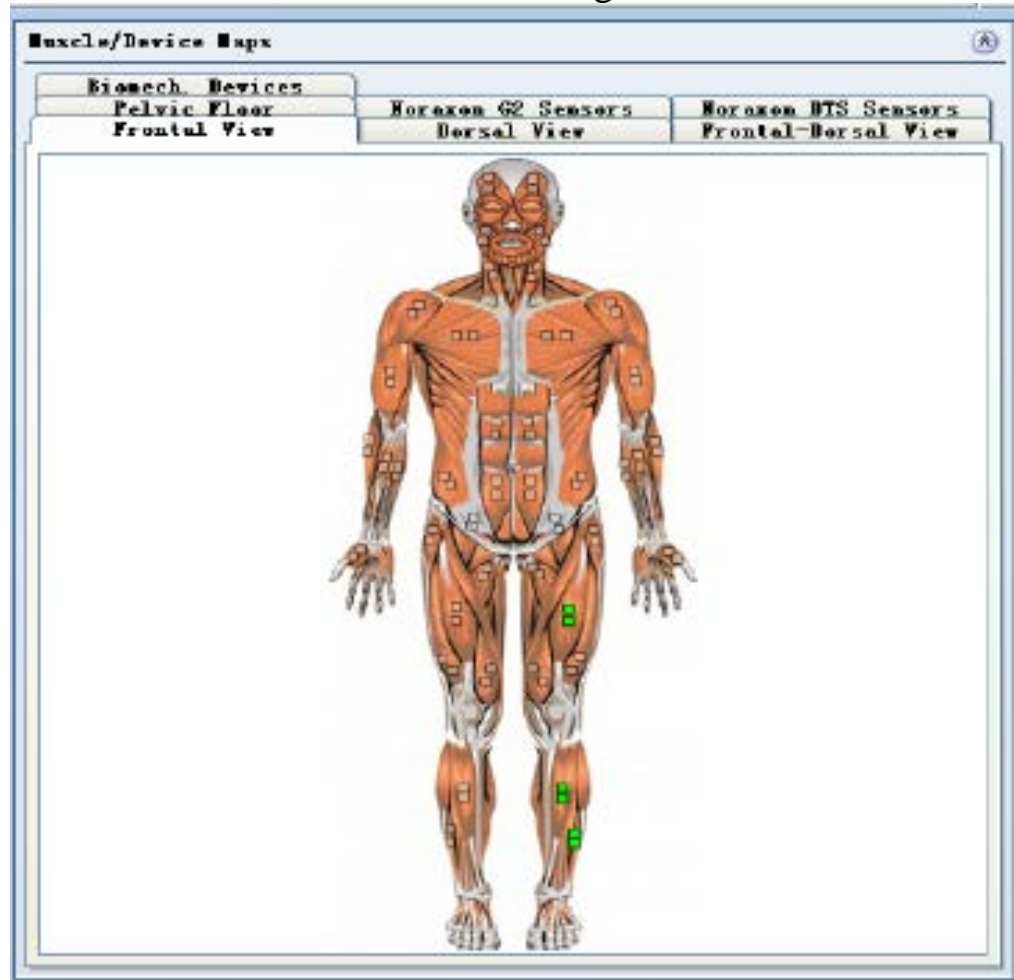

Fig. 3 Schematic diagram of EMG analysis 3D interface

Figure 3 shows the EMG software MyoResearch XP with professional equipment. The software is the most complete EMG and biomechanical transducer software currently on the market [10]. The software can do the analytic design of 3D motion [11-12]. The movement decomposition process is as shown in Figure 4.

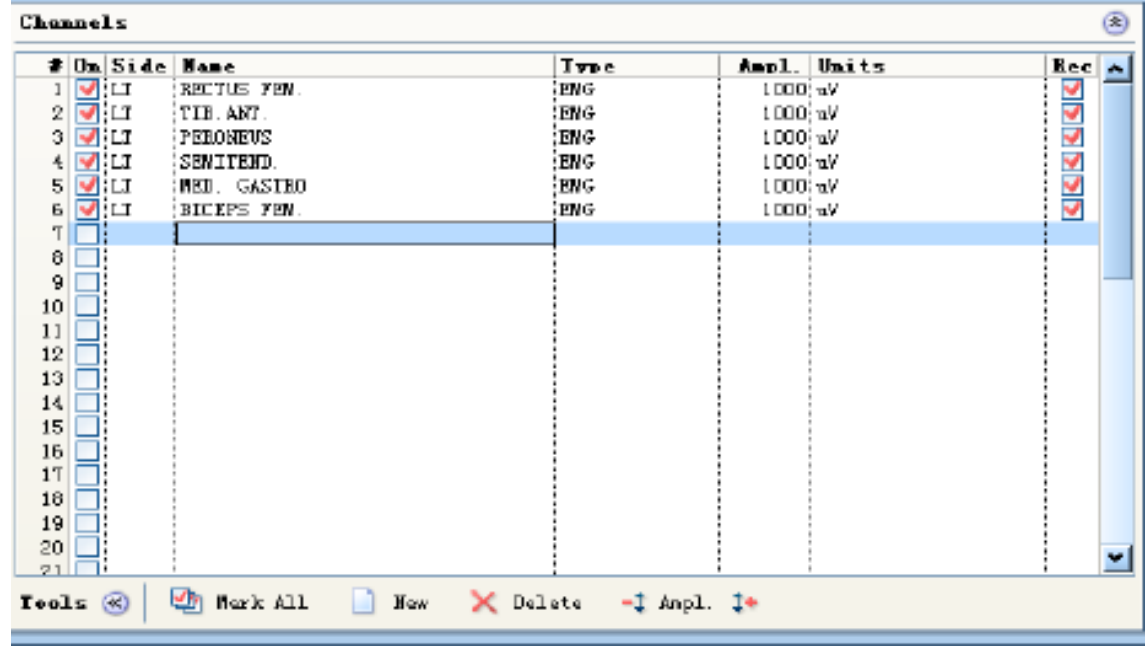

Fig. 4 Schematic diagram of EMG image analysis process 
Figure 4 shows the schematic diagram of 3D motion analysis process. Different from the general software, it has many tools suitable for complex science research. The more important is it has numerous reports to be called for users $[13,14]$. The software has high intelligent degree, and it can show what kinds of data to collect, how to deal with and analyze the calculation etc..

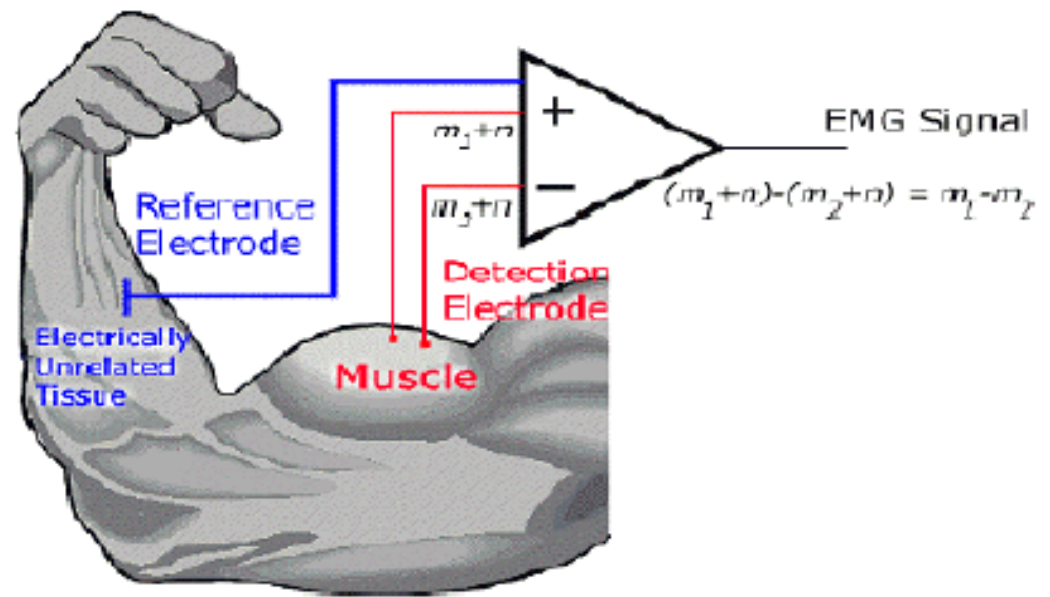

Fig.5: Schematic diagram of electrode paste

As shown in Figure 5, in the EMG test, sensor train gauge needs to paste in electrically neutral organization, usually near the bone, and as far away as possible from the recording electrode. The selected position should meet the large size, good adhesive contact to ensure better electrode contact between the reference electrode and the tissue.
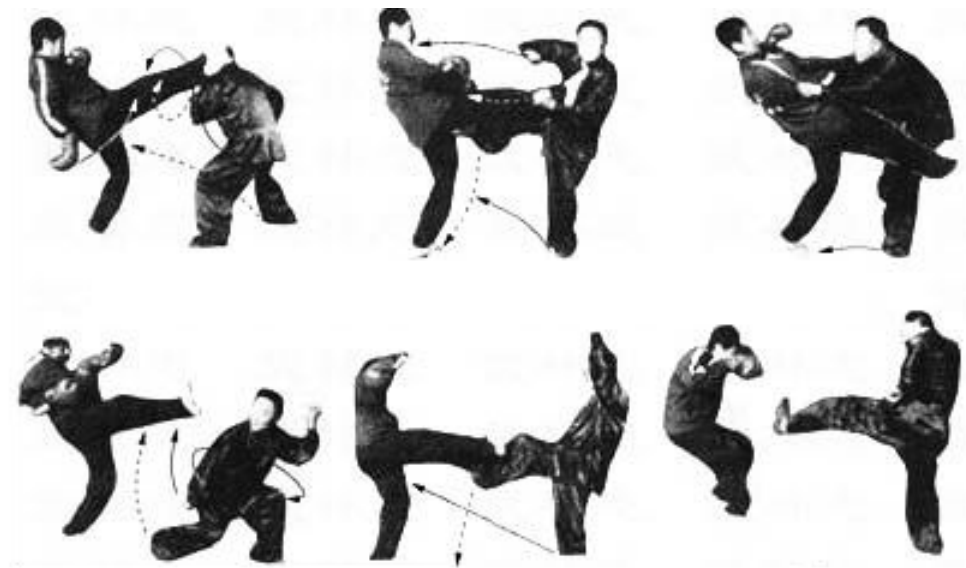

Fig.6: Wushu continuous action images

Figure 6 represents a group of martial arts action image in this paper. The image is divided into 8 movements, each motion has 3D move, and 8 moves are coherent [15, 16]. Through repeated training, the variation curve of the reaction time is obtained, as shown in Figure 7.

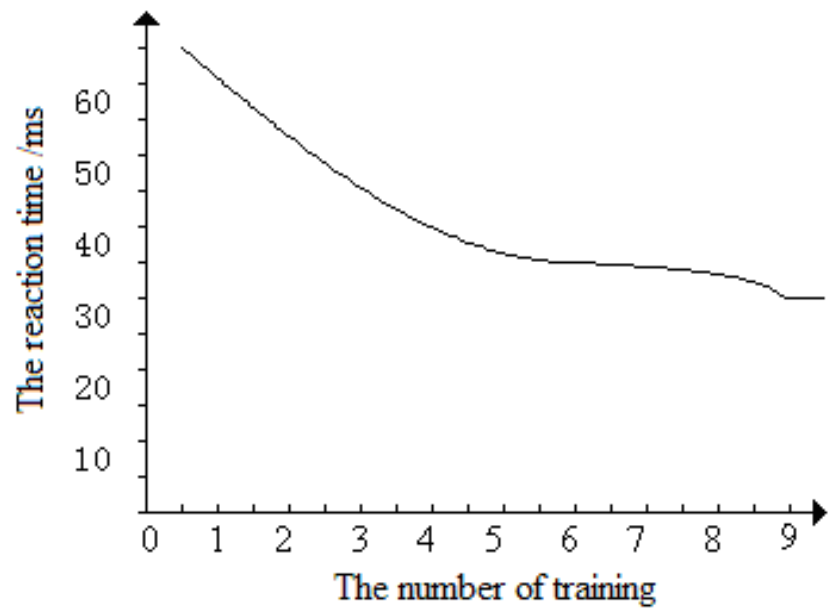

Fig.7: Training number response curve changing with time 
Figure 7 shows the training number response curve changing with time. From the chart, it can be seen, with the increase of training times, the reaction time of limb movement is more and more short, reaction action is more and more quickly $[17,18]$. When reaching to the sixth time, the reaction time has been gradually stabilized.

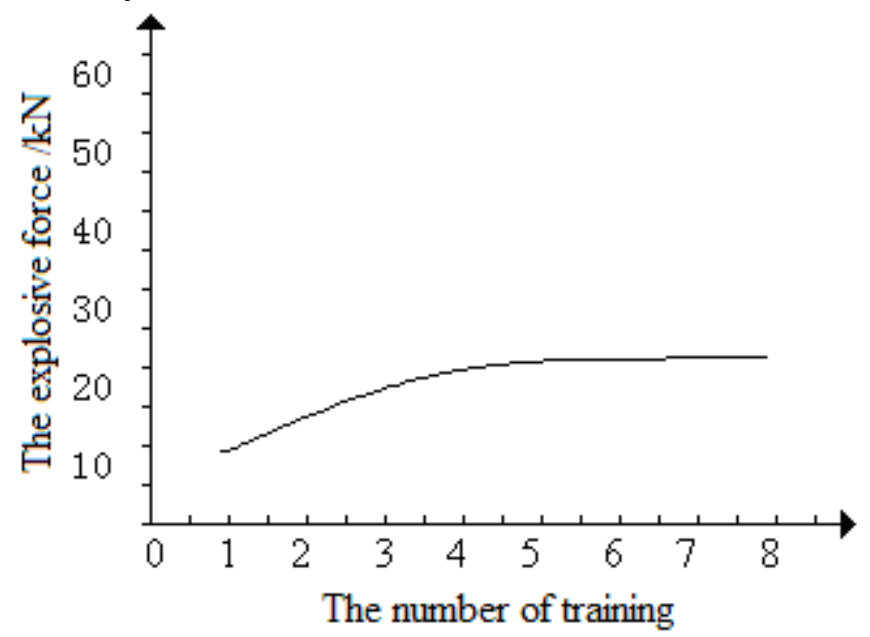

Fig.8: Biological explosive force curve changing with the training number

Figure 8 represents biological explosive force curve changing with the training number. From the chart it can be seen, with the increase of training times, body movements explosive force is more and more big [19, 20]. When reaching the fifth times, the explosive force is stabilized. And the explosive force and reaction time is basically collaborative, which achieves good optimization effect of free fight techniques.

\section{Summary}

On the basis of relationship matrix and logical path mathematical model, this paper proposes a new 3D image analysis method, and this method can do EMG analysis on free fight body movement reaction speed and explosive force, which improves the precision of image analysis. In order to verify the effectiveness and the reliability of the algorithm, this paper uses MyoResearch XP to test and analyze the EMG. Through the analysis of free fight techniques reaction speed and biological explosive force, this paper obtains the synergy curve of physical reaction speed and explosive force, which provides the theory reference for the study of EMG image analysis technology.

\section{References}

[1] S.Y. Ma. Analysis of Taekwondo starting operation. Contemporary sports science and technology, 2012, 5(3): 45-48.

[2] Y.F. Li, Cao. The response levels of Adolescent tennis athletes. Hubei sports science and technology, 2011, 2(6): 117-119.

[3] X.P. Chen. Research on the factors of affecting the reaction rate and training method. Sports scientific literature review, 2011, 5(3): 89-90.

[4] Y.P. Li, Q.J. Pang. Research on the effects factor of the athletes' reaction velocity. Science and technology for development, 2011, 2(2): 126-129.

[5] J. Miao, X.D. Zhang, F. Yu. Discussion on the Sanshou coaches on the development of athletic psychological ability. Fight, 2011, 3(1): 23-26.

[6] D.L. Zhang. Comparative study of Sprinters' start reaction time at the twenty-ninth Olympic Games. Journal of Zhaoqing University, 2012, 1(5): 78-80.

[7] J.M. Zheng, Z.J. Chen. The fast interactive Graph-Cut algorithm with the connectivity constraint. Journal of computer aided design and graphics, 2011, 4(3): 78-80.

[8] S.D. Han, Y. Zhao, W.B. Tao. The fast Graph Cuts image segmentation based on Gauss super pixel method. Journal of automation, 2011, 4(5): 154-155. 
[9] S.Q. Li, Q.J. Feng. The unified compute device architecture parallel graph cut algorithm used by liver tumor image segmentation. Journal of Chinese biomedical engineering, 2011, 2(5): 103106.

[10] C. Liu, F.X. Li, Y. Zhang. The objects segmentation method based on graph cut and pan shape information. Journal of Computer Aided Design \& computer graphics, 2011, 5(12): 975-984.

[11] Z.W. Yu, Z.Y. Yu, X.S. Zhou. Social aware computing: concepts, issues and research progress. Chinese Journal of computers, 2012, 5(3): 91-95.

[12] J.X. Wang, J.Q. Ni, J.W. Pan. A high performance of reversible watermarking algorithm based on histogram shifting. Journal of automation, 2012, 6(2): 136-140.

[13] H.S. Zhu, W. Wang, B.L. Shi. The frequent closed plot and its generating No redundant plot rule extraction. Journal of computer, 2012, 7(1): 81-84.

[14] T. Tang, Y.S. Lin, X. Yang. CPU-GPU heterogeneous system comprehensive energy consumption optimization method based on critical path analysis. Chinese Journal of computers, 2012, 4(1): 136-140.

[15] X.J. Lei, J.F. Tian. The colony information flow clustering model and algorithm of protein interaction networks. Journal of computer of protein interaction, 2012, 6(1): 56-59.

[16] L.X. Liu, Z.M. Ma, H.B. Zhao. A segmentation method of cardiac magnetic resonance images based on active contour model. Journal of computer, 2012, 5(1): 23-26.

[17] Q. Fu. Characteristics study of high level fencing visual reaction time. Journal of Capital Institute of Physical Education, 2012, 2(5): 34-37.

[18] Q.P. Sun, L. Gao. Study of our country excellent men Sanshou athletes' sport cognitive trait anxiety. Shandong sports science and technology, 2012, 3(30): 45-48.

[19] Y.G. Ceng. Study of Sanshou athletes' reaction speed and method. Tsinghua Science and technology, 2012, 4(1): 24-27.

[20] Y. Chang. Comparative study of adolescent Sanshou athletes and adult Sanshou athletes reaction. Fight (Wushu science), 2012, 3(4): 56-59. 